\title{
AUV Abyss workflow: autonomous deep sea exploration for ocean research
}

\author{
Meike Klischies \\ Magmatic and Hydrothermal Systems \\ GEOMAR - Helmholtz Centre for \\ Ocean Research Kiel \\ Kiel, Germany \\ mklischies@geomar.de \\ Isobel A. Yeo \\ Marine Geoscience \\ National Oceanography Center \\ Southampton, UK \\ i.yeo@noc.ac.uk \\ Claas Faber \\ Data Centre \\ GEOMAR - Helmholtz Centre for \\ Ocean Research Kiel \\ Kiel, Germany \\ cfaber@geomar.de
}

\author{
Marcel Rothenbeck \\ Magmatic and Hydrothermal Systems \\ GEOMAR - Helmholtz Centre for \\ Ocean Research Kiel \\ Kiel, Germany \\ mrothenbeck@geomar.de \\ Christian dos Santos Ferreira \\ Marine Geology \\ MARUM - Center for Marine \\ Environmental Sciences \\ Bremen, Germany \\ cferreira@marum.de \\ Carsten Schirnick \\ Data Centre \\ GEOMAR - Helmholtz Centre for \\ Ocean Research Kiel \\ Kiel, Germany \\ cschirnick@geomar.de
}

\author{
Anja Steinführer \\ Magmatic and Hydrothermal Systems \\ GEOMAR - Helmholtz Centre for \\ Ocean Research Kiel \\ Kiel, Germany \\ asteinfuehrer@geomar.de \\ Jochen Mohrmann \\ Marine Geosystems \\ GEOMAR - Helmholtz Centre for \\ Ocean Research Kiel \\ Kiel, Germany \\ jmohrmann@geomar.de
}

\begin{abstract}
Autonomous underwater vehicles (AUVs) equipped with multibeam echosounders (MBES) are essential for collecting high-resolution bathymetric data in the deep sea. Navigation of AUVs and accuracy of acquired MBES data is challenging, especially in deep water or rough terrain.

Here, we present the AUV Abyss operational workflow that uses mission planning together with a long baseline (LBL) positioning network, and systematic post-processing of the MBES data using feature matching. The workflow enables autonomous exploration even in difficult terrain, makes ultrashort baseline navigation during the AUV survey obsolete and with this, increases the efficiency of ship time. It provides an efficient workflow for multi-survey mapping campaigns to produce high-resolution, large-coverage seafloor maps. Automated documentation of post-processing steps enhances the archiving of produced results, facilitates knowledge transfer, adaptation to other systems and management of large datasets. Comprehensive documentation allows developing routines that provide a first step towards automatization of AUV operations and MBES data processing.
\end{abstract}

Keywords- AUV navigation, high-resolution mapping, dive planning, post-processing, multi-survey campaigns

\section{INTRODUCTION}

The AUV Abyss is primarily used to collect hydroacoustic datasets in deep water over rough terrain. In this paper we present AUV Abyss workflow, which has been established over nearly a decade of deep sea exploration including multi-survey mapping campaigns in rough, deep sea environment producing large-coverage, high-resolution seafloor maps.

The operational workflow describes best practice mission planning and works without ultra-short baseline navigation (USBL) and minimizes the reliance on long baseline (LBL) positioning, which is commonly degraded by the presence of steep cliffs and valleys. The corresponding data processing workflow maximizes documentation, archiving and knowledge transfer.

The AUV Abyss is a torpedo-shaped Remus 6000 type AUV (built by Hydroid, LLC, USA). The system was bought by the German Research Foundation (Deutsche Forschungsgemeinschaft, DFG) and has been operated by GEOMAR - Helmholtz Centre for Ocean Research Kiel since 2008 [1]. Since then, the AUV Abyss has conducted over 300 dives and the seafloor maps produces have been featured in numerous publications, e.g. [2]-[4].

The AUV Abyss workflow combines careful mission planning with systematic post-processing. The first part of this paper summarizes the complex mission planning process and provides best practice solutions. The second part of the workflow describes the post-processing of MBES data and corresponding map production, data management, and documentation.

Furthermore, this paper discusses associated uncertainties and future developments in terms of adaptation and automatization of the workflow.

\section{A. AUV Abyss Specifications}

Besides the AUV Abyss specifications summarized in table 1, the system also comprises a control and workshop container, and a mobile Launch and Recovery System (LARS; developed by WHOI) with a deployment frame to be installed at the stern of the afterdeck or the side of an oceangoing research vessel (Fig.1A). The LARS allows AUV launch and recovery at weather conditions with a swell up to $2.5 \mathrm{~m}$ and wind speeds of up to six Beaufort.

\section{B. Fields of Use}

The AUV Abyss can be operated in water depths up to $6,000 \mathrm{~m}$, which makes it applicable in the vast majority of Earth's ocean. GEOMAR's research activities target a huge variety of submarine environments including mid-ocean ridges, seamounts, fracture zones and back-arc basins. In this rough terrain, AUV Abyss operations aim for highresolution, large-coverage mapping to enable scientific interpretation of the seafloor, mapping of small features and characterization of habitats. The AUV Abyss workflow has to allow adjustments to complex terrain including cliffs, 
calderas, slopes and volcanically active areas with variable seafloor properties.

TABLE I. AUV ABYSS TECHNICAL DETAILS

\begin{tabular}{|c|c|}
\hline Item & Specifications \\
\hline Vehicle dimensions & Length: $4.10 \mathrm{~m}$; diameter: $0.66 \mathrm{~m}$ \\
\hline Weight in air & $880 \mathrm{~kg}$ \\
\hline Depth rating & $6000 \mathrm{~m}$ \\
\hline Survey speed & $3 \mathrm{kn}$ \\
\hline Endurance & 15-23h (depends on used sensors) \\
\hline Power source & Lithium-Ion battery \\
\hline MBES & RESON Seabat $7125(200 / 400 \mathrm{kHz})$ \\
\hline Sidescan sonar & Edgetech 2200-S (120/410kHz) \\
\hline LBL system & $\begin{array}{l}\text { Hydroid transponders }(6000 \mathrm{~m}, 4 \text { channel, } \\
9-13 \mathrm{kHz})\end{array}$ \\
\hline Vehicle sensors & $\begin{array}{l}\text { Kearfott T-24 INS; Teledyne RDI } \\
\text { Workhorse Navigator DVL WHN 300; } \\
\text { Seabird CTD SBE49 FastCat; Wetlabs } \\
\text { FLNTURTD 939 Turbidity Sensor; Eh } \\
\text { Sensor (Ko-ichi Nakamura); Applied } \\
\text { Physics Magnetometer Model 1540 }\end{array}$ \\
\hline Propulsion & $\begin{array}{l}\text { Three-phase brushless DC motor; two } \\
\text { blade propeller }\end{array}$ \\
\hline
\end{tabular}

\section{MisSION PLANNING}

Mission planning comprises survey setup, survey design and the LBL network setup. The research question determines the most essential factors to consider: applied sensor configuration, sensor setup, ideal resolution and targeted area. The following mission planning workflow is applicable for the MBES configuration, which is the most common AUV Abyss operation setup.

Pre-existing information is gathered in a geographic information system (GIS) project using software packages such as GlobalMapper (by Blue Marble Geographics) or QGIS (www.qgis.org). In this GIS environment, the survey setup is planned (section IIA), the LBL network (IIB) positions and the AUV survey design (IIC) are geographically plotted. If available, MBES settings are adjusted to known seafloor substrate properties (IID). In a final step, the mission planning is translated into a scripted mission file to be read by the AUV Abyss (IIE).

\section{A. Survey Setup}

Both, the desired resolution (and therefore the operating frequency of the system) and the targeted area define the survey setup; meaning, (i) is a single survey sufficient or a multi-survey campaign required to cover the targeted area, and (ii) is an LBL network needed to provide an initial position update to correct for drift acquired during the descending phase.

An individual survey is limited in coverage by dive time, which is controlled by battery capacity. Better coverage can be achieved by surveying at a higher survey altitude, however, this lowers the resolution of the acquired MBES data. An individual AUV Abyss mission maps on average $10 \mathrm{~km} 2$ in a spatial resolution of $3 \mathrm{~m}$, when it surveys at $3 \mathrm{kn}$ and $80-100 \mathrm{~m}$ altitude with standard MBES settings (see IID).

A multi-survey campaign is needed if the targeted area exceeds the mapping capability of an individual mission (Fig. 1B). To facilitate corresponding merging of multiple surveys during the post-processing, outer tracks of adjacent missions should overlap to $100 \%$.

\section{B. LBL Network}

Both, the desired resolution (and therefore the operating frequency of the system) and the targeted area define the survey setup; meaning, (i) is a single survey sufficient or a multi-survey campaign required to cover the targeted area, and (ii) is an LBL network needed to provide an initial position update to correct for drift acquired during the descending phase.

An individual survey is limited in coverage by dive time, which is controlled by battery capacity. Better coverage can be achieved by surveying at a higher survey altitude, however, this lowers the resolution of the acquired MBES data. An individual AUV Abyss mission maps on average $10 \mathrm{~km} 2$ in a spatial resolution of $3 \mathrm{~m}$, when it surveys at $3 \mathrm{kn}$ and 80-100m altitude with standard MBES settings (see IID).

A multi-survey campaign is needed if the targeted area exceeds the mapping capability of an individual mission (Fig. 1B). To facilitate corresponding merging of multiple surveys during the post-processing, outer tracks of adjacent missions should overlap to $100 \%$.

\section{Survey Design}

Careful planning of the AUV mission track is the most crucial part, as it must maintain a constant doppler velocity $\log$ (DVL) bottom detection throughout the entire survey to ensure accurate positioning. Further, mission track planning balances survey setup, aimed resolution and terrain, but also considers MBES settings, and demands for efficient data post-processing and map generation.

Survey design relies on pre-existing terrain data of the area. Terrain data with a minimum resolution of $100 \mathrm{~m}$ proofed as sufficient and must be collected before the AUV surveys.

Terrain, desired resolution and AUV MBES specifications (e.g. range settings, see IID) determine the overall mission track altitude; e.g. AUV Abyss mapping at $70 \mathrm{~m}$ above seafloor commonly results in a final grid resolution of $2 \mathrm{~m}$.

The AUV Abyss can map in two different altitude modes (Fig. 1C): either (i) at constant water depth coinciding with variable altitude and hence variable resolution, or (ii) at a constant altitude resulting in constant MBES data resolution, but also requiring the vehicle to pitch more during the survey. A constant water depth ensures a smoothly moving vehicle and constant MBES data quality, if the terrain varies within the range of the MBES. Larger altitude changes require adjusting MBES range settings; therefore, the constant altitude is usually preferred.

The mission track pattern depends on the dimensions of the target area, corresponding terrain and the post-processing demands. A lawn-mower pattern, ideally with long, continuous survey lines generally produces the best data, as it reduces vehicle movements while simultaneously covering large areas (Fig. 1B). Over the duration of a survey, the vehicle drift increases; therefore, mission planning should avoid large time differences between overlapping, neighbouring or crossing tracks to ensure good, relative positioning and sufficient overlap.

The orientation of the survey lines will ideally follow the terrain to prevent crashes or losing DVL bottom lock. A mission track pattern parallel to contour lines also reduces 
pitch changes, which is especially important for the constant altitude mode. Slopes are best mapped contour-parallel from top to bottom. Steeper terrain requires more tightly-spaced survey lines.

The spacing of survey lines has to ensure a consistent map coverage. Ideally, the line spacing causes an MBES swath overlap of at least $25 \%$, and of $50 \%$ at best (Fig. 1D). This also makes the navigation adjustment process using the feature matching algorithm much easier.

Navigation adjustment of the data is further improved by including tie lines in the mission track planning. Tie lines cross the lawn-mower pattern at an angle with full overlap (Fig. 1B) and with this, provide additional spatial reference.

Multi-survey campaigns require merging of data sets during post-processing; therefore, mission track planning for multi-survey campaigns has to ensure sufficient overlap in adjacent survey areas. Overlaps should be total, meaning $100 \%$, for the outer one or two tracks of each survey. Tie lines that cross other survey areas can further improve the navigation adjustment and merging result.

If an LBL network is used for an initial position fix, the mission track planning has to enable both, DVL bottom detection and triangulation of the position fix with the LBL network. The usual LBL network setup outside of the target area (see section IB) results in a transit line towards the mapping area that can act as an additional tie line.

\section{MBES Settings}

The AUV Abyss is equipped with a RESON Seabat 7125 MBES working at 200 and $400 \mathrm{kHz}$. The MBES opening angle is fixed at $128^{\circ}$ angle tightening the mission track planning (see section IC). The AUV transmits internal navigation, vehicle attitude data and sound velocity information with more than $8 \mathrm{~Hz}$ to the MBES. Its processing unit saves these records together with the sounding data into the raw s7k-files.

MBES settings to be adjusted in each survey include logging modes (on/off), power, pulse length, gain, range and ping rate. Power, gain and pulse length are set according to available substrate information (e.g. seafloor sampling) or based on MBES performance in initial surveys. The range, in which the sonar aims to detect the seafloor, depends on the survey altitude. The ping rate is set to maximum and with this, automatically adjusted to the range setting.

MBES settings are defined in the objectives section of the mission file.

\section{E. Mission File}

The mission file is a ASCII (text) file using humanreadable commands that transmit the survey planning to the AUV Abyss. The mission file is separated into a reference position section and an objectives section. The reference position section defines the LBL transponder positions the AUV Abyss needs for triangulation of its position prior to surveying, and positions of internal reference points.

The objectives section is the main part of the mission file. It sets waypoints and associated speed, dive mode (attitude/altitude) and MBES settings to be applied (see above, IID). Enabling and disabling of LBL positioning is also set in this section, but is usually restricted to the early phase of a mission: after the first DVL lock and before the MBES survey start to prevent position updated during the MBES survey.

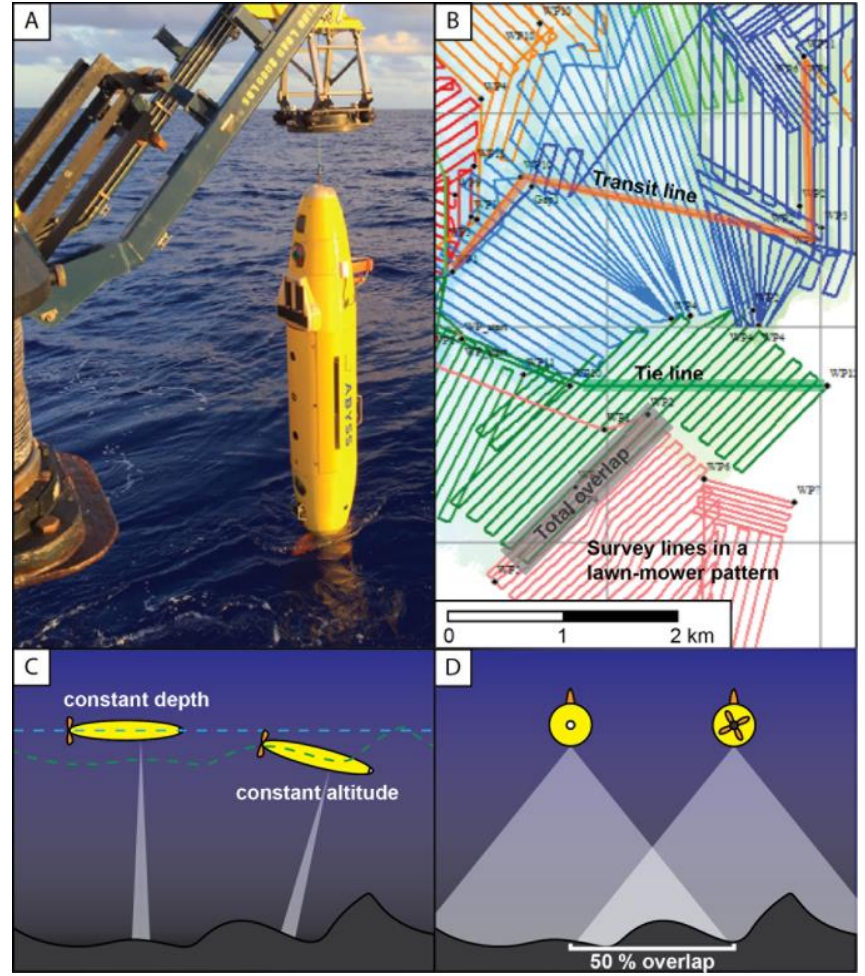

Fig. 1. A) AUV Abyss in the MBES configuration during recovery using the LARS on board R/V Meteor in 2016 (cruise M127 [5]). B) Part of the mission pattern of the multi-survey campaign successfully conducted during cruise M127, showing mission tracks of $200 \mathrm{kHz}$ MBES surveys including lawn-mower patterns, total overlap for merging of adjacent surveys, transit lines between the LBL navigational gate and the survey area, and tie lines for additional navigational constrains (modified from [5]). C) Sketch showing the two different mapping modes. The constant depth mode results in stable vehicle motion, but variable swath width and MBES data resolution. The constant altitude mode maps in a constant resolution and swath width, but rough terrain causes vehicle motion and variable coverage. D) Sketch illustrating overlap in MBES swath data between neighbouring survey lines.

\section{Post-Processing AND NAVIGATION AdJUSTMENT}

The post-processing workflow is based on the open source software distributions MB-System [6], Python [7], and Jupyter [8] and is distributed as Jupyter Notebooks (section IIIA). The Jupyter Notebooks guide and facilitate the documentation of the post-processing workflow, corresponding obstacles and applied solutions. With this, the notebooks act as a metadata file of how the post-processing was conducted. They also assist data management including data handling, documentation and archiving of generated products (IIIB).

The plus-side of working in rough terrain is that it presents plenty of opportunity for using feature matching in MBES data to adjust the vehicle track and correct for drift (IIIC), and to merge adjacent, overlapping surveys (IIID). In the very last step, the (navigation adjusted) MBES sonar data is manually cleaned from outliers and erroneous data and then gridded to produce a final bathymetric grid (IIIE).

\section{A. Software}

The workflow is published as Jupyter Notebooks running on MacOS and Linux operating systems with the open source distributions MB-System [6] (version 5.5.23x), Python [7] (version 3.x), and Jupyter [8] (version 5.0.0) installed. 
MB-System is an open source software package comprising a highly diverse collection of tools for processing and analyzing swath mapping sonar data in a large variety of formats. MB-System is used in conjunction with Generic Mapping Tools (GMT, [9]), an open source collection of tools for editing geographic and Cartesian data sets, producing and plotting maps.

Packaged MB-System distributions contain GMT and other prerequisites, and are available for MacOS (as a Homebrew package) and Linux operating systems (included in Poseidon Linux and maintained in UbuntuGIS).

Python is an open source programming language used to integrate a variety of tasks into the workflow that work beyond MB-System capabilities. Python is available in several packages and distributions, of which the Anaconda Distribution (www.anaconda.com) also includes Jupyter Notebook.

Jupyter Notebook is an open source web application for interactive computing across programming languages that combines live coding, visualization, narrative text, and output documentation. The notebooks act as a wiki combined with a command line terminal allowing users to run commands through a web interface and automatically redirecting and archiving the terminal standard output into the notebook. With this, the notebooks facilitate sharing, adaptation, execution, documentation and archiving of AUV Abyss MBES post-processing.

Maintaining a GIS project parallel to the post-processing workflow is recommended to keep an overview, check intermediate results and produced digital elevation models. This is especially applicable to surveys, where the DVL detection was lost, and to multi-survey mapping campaigns, where post-processing has to manage and merge several surveys. GIS projects allow to compare the AUV data to ship based bathymetry or other available terrain data and to approximate survey positions. With this approximation, the corresponding data can be manually shifted to make navigation adjustment possible.

\section{B. Data Management}

To allow structured collaborative development and versioning, the Jupyter Notebooks are hosted on a public Git repository [11]. Publishing notebooks on the GEOMAR Git server provides public access via well-known standards, i.e. git checkout or download of an archive file. Contributions by external parties and structured development processes are well supported and documented via merge requests and issues.

To ensure long-term availability, a Digital Object Identifier (DOI) has been assigned to the version of the notebooks presented in this publication [10]. The DOI resolver directs to the landing page providing not only the notebooks themselves, but also links to the cited and future versions of the notebooks in the Git repository.

The AUV Abyss workflow is designed to use raw MBES data, which is translated into an MB-System-specific format. Each processing step creates copies of the data, instead of editing the existing files. This implemented back-up provides the opportunity to redo individual steps in case of obstacles rather than redoing the entire workflow. Together with auxiliary files that are created automatically by MB-System, the amount of files increases rapidly over the course of a project. This makes a robust data management structure and naming convention inevitable. MB-System uses datalists to organize the project data and the AUV Abyss Jupyter
Notebook includes both, executable python code and narrative comments, to systematically maintain the data management structure.

The final products comprise both ascii grid files and asci text files of the point cloud sounding data. The grid files are used to check the navigation adjustment results, while the text files (xyz-format) are the export product containing the latitude, longitude and depth information of the navigation adjusted sonar data. Export of the adjusted vehicle track is also possible.

Automated archiving stores the raw MBES data, the adjusted MBES data, and the navigation adjusted and cleaned data. It also includes selected auxiliary files, such as those created by the tool mbnavadjust (see below).

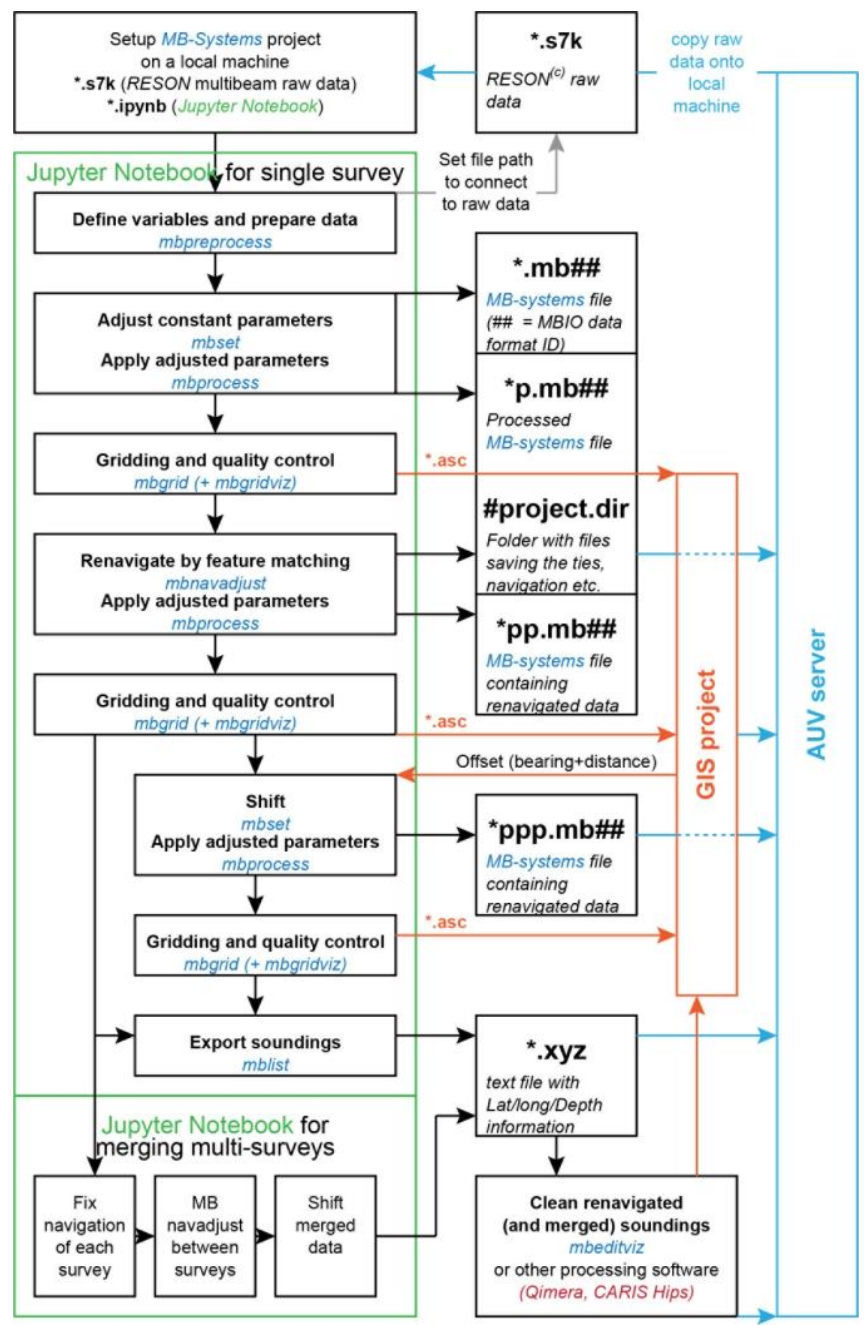

Fig. 2. Schematic overview of the AUV Abyss post-processing workflow for MBES data and corresponding data management.

\section{Navigation Adjustment}

Figure 2 gives a schematic overview of the individual processing steps that mainly use the MB-System tools mbpreprocess, mbset, mbprocess, and mbnavadjust.

Mbpreprocess prepares the raw data for processing by converting it into an MB-System-specific format. Mbset is used to generate a default parameter file for each raw file and set its values. This includes vehicle specific parameters (e.g. lever arm offsets) and mission specific parameters (e.g. horizontal shifts to correct for drift). Mbprocess applies edits made in the parameter files, and can perform a variety of 
other processing functions. Mbnavadjust, the 'multibeam navigation adjustment' tool, is the most crucial part of the AUV Abyss workflow, as it eliminates relative navigational errors. The program uses bathymetric feature matching in overlapping and crossing swaths to invert the corresponding navigation (Fig. 3 A-B). For this, the swath sonar data is automatically split into tiles of a user defined length, in which the relative shift of bathymetric features is manually corrected by matching contour lines of overlapping tiles.

Mbnavadjust works through an interactive graphical interface that is started from the Jupyter Notebook. Comments in the notebook provide best practice descriptions guiding through the navigation adjustment. The feature matching of the AUV Abyss workflow is conducted in consecutive steps. Starting from tiles with true crossings (total overlap), features are matched in a second and third step in tile-pairs with $50 \%$ and $25 \%$ overlap, respectively. After each matching step, the navigation is inverted (by minimizing the first derivative of the perturbation following e.g. [12]; [6]) and applied to the data.

If no positional fixes are available, the navigation adjusted data set is compared to pre-existing terrain data, and manual correction for drift, hence horizontal shift, is applied. The drift is estimated from bathymetric features in the navigation adjusted grid and pre-existing terrain model data, preferentially the GPS-navigated ship-based bathymetry. Mbset assigns the shift in the parameter file and mbprocess implements the shift into the navigation adjusted sonar data.

Shift of data sets relative to surveys with a good, robust navigation can facilitate the merging process, as it provides more accurate overlap. This is also applicable to surveys that experienced DVL loss. In case of shifting relative to ship's bathymetry, the resulting accuracy of the AUV map grid equals that of the underlying ship data.

\section{Merging Multiple Surveys}

The second part of the post-processing only applies to multi-survey campaigns as it merges MBES data of adjacent, overlapping surveys. It uses two different approaches, depending on the previous treatment of individual surveys.

In cases where the individual surveys had to be shifted (see above), the internal navigation of each individual survey has to be fixed, before merging it with others. This is achieved by setting navigational ties (every third or fourth) within the $25 \%$ overlap tiles of each mission in a new mbnavadjust project. Subsequently, overlapping tiles of two different surveys are matched (Fig. 3 C-D).

In case of unshifted surveys, the existing mbnavadjust projects can be merged by using the tool mbnavadjustmerge. This approach translates the already set ties of each individual survey into a new merge project, but requires sufficient overlap in the adjacent surveys.

Although the first approach repeats setting of ties, it prevents the relative contraction of the data by correcting for drift (shift of data) prior to merging. Contraction of data is usually observed in very large, merged datasets compiling several surveys over extensive areas.

\section{E. Data Cleaning}

Manual cleaning of outliers in MBES data is the last step of the AUV Abyss workflow; although, appropriate usage of the mbnavadjust tool might require some filtering and cleaning (e.g. using mbedit or mbeditviz) prior to the navigation adjustment.
As the cleaning process is very labour intensive, it's efficiency also depends on the operator's preference in software. Besides using the MB-System tools mbeditviz and mbnavedit, the Jupyter Notebooks also allow exporting the navigation adjusted, shifted and/or merged data in arbitrary formats (using mblist and mbnavlist commands) to be used for cleaning and gridding the data in other processing software, e.g. Qimera (QPS) or CARIS HIPS (Caris), instead of MB-System.

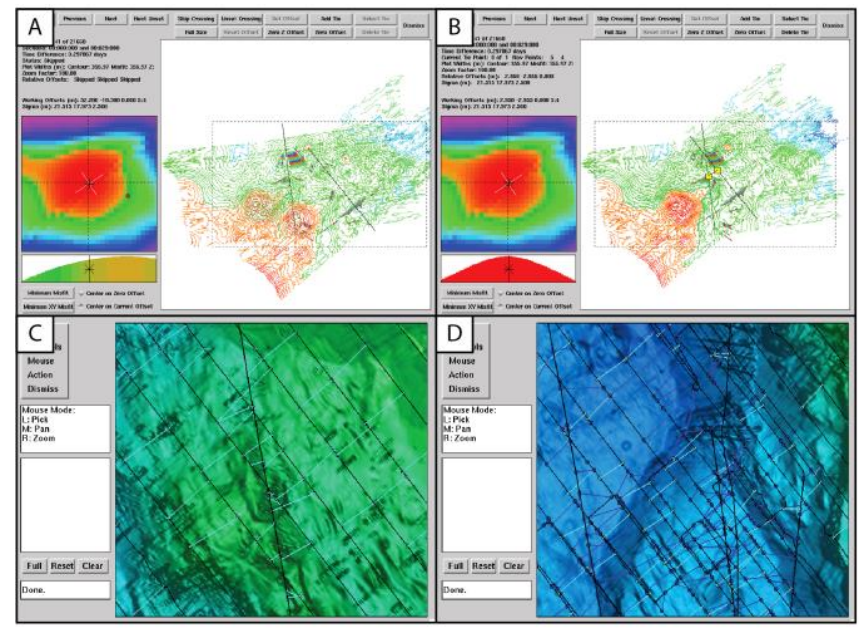

Fig. 3. A) Mbnavadjust 'Nav Err Window' to inspect colour-shaded contour lines of two tiles of a true crossing (dotted rectangle) and corresponding mission tracks (black lines) in the main 'Contour Display'. The 'Vertical Misfit Display' in the lower left corner shows the RMS bathymetric misfit as a function of relative horizontal offset between the two swaths. B) 'Nav Err Window' showing feature matched tiles (low misfit, marked by red in the 'Vertical Misfit Display') and a set tie (connected yellow squares). C) Mbnavadjust 'Visualize Survey' plot with mission tracks (black lines) and set ties (light blue lines) of an individual survey with a tie line. D) 'Visualize Survey' plot of a merged, multi-survey campaign with set ties within each individual survey (light blue lines) and set ties in between surveys (dark blue lines). Shown AUV Abyss data was acquired during the BGR INDEX project (INDEX2016-2).

\section{DISCUSSION}

The presented operational workflow works without USBL navigation and instead, uses an LBL network for an initial position fix. Robust USBL positioning during the descent of the vehicle has the potential to replace the initial position fix obtained with the LBL network. But, as both, calibrating the LBL network and communicating with the descending vehicle via USBL, cost similar ship time, USBL positioning is only really a good option for single surveys. An LBL network is the preferred option during multi-survey mapping campaigns, as it saves ship time.

Adaptation of the mission planning workflow to other AUVs depends on the vehicle type and their setup. Nevertheless, mission tracks producing sufficient overlap in the MBES data remain crucial, when aiming for highresolution, large-coverage maps of the seafloor.

The presented post-processing approach aims to reduce uncertainties in data handling and producing high-resolution, large-coverage maps of the deep sea. Remaining uncertainties mainly include the unknown terrain itself, but also the objectivity of the interpreter in feature matching. Profound knowledge of the survey terrain ensures both, a solid survey planning and robust feature matching. 
Feature matching and corresponding navigation adjustment is facilitated by MBES data of good quality and data sets are ideally cleaned prior to the navigation adjustment. This limits the cleaning to MB-System tools, or requires an extra step of exporting and importing data for cleaning in other processing software. Therefore, the presented workflow aims to ensure good data quality through careful mission planning, and neglects tiles with poor data in the navigation adjustment. If necessary, application of filters (e.g. using mbedit) proved as sufficient for a robust navigation adjustment.

The systematic, guided post-processing approach addresses the objectivity of the feature matching. Although the feature matching remains subjective to a certain extent, the use of Jupyter Notebooks and coded archiving increases the reproducibility of results. The implemented back-up of intermediate results proved as important for learning and becomes obsolete with increasing post-processing experience and routine.

Adaptation and reproducibility are further supported by using only open source software distributions. MB-System supports more than four-dozen formats from sonar equipment manufactured and operated around the world [6]. The mbnavadjust tool also enables navigation adjustment and merging across formats and resolutions.

With this, the presented post-processing workflow is adaptable to other AUVs and MBES setups, but also applicable for multiple coordinated AUVs. The mbnavadjust tool restricts the application of this workflow to mapping of rough, feature-rich terrain, and MBES data of good quality and sufficient overlap.

Despite the systematic approach, post-processing of AUV Abyss MBES data remains time consuming; therefore, future objectives aim to automatize the workflow and to establish web-based co-working possibilities. Building the workflow upon the web-based approach of Jupyter Notebooks will allow remote access and enable multiple coworkers on the same project in the future.

The mbnavadjust tool provides a function for automatic feature matching, but the results are limited in complex terrain. The manual approach proved as more efficient. Automatized feature matching is also implemented in SLAM-like mapping techniques (Simultaneous Localization and Mapping, [13]). The survey-simultaneous localization is desirable, but not applicable to the current AUV Abyss vehicle setup. Nevertheless, SLAM algorithms have the potential to advance and automatize the post-processing of MBES data in the future.

Although a fully automated post-processing workflow would not be based on Jupyter Notebooks, they document todays necessities in post-processing, according obstacles and valid solutions. With this comprehensive documentation, they aid to establish post-processing routines and systematic data handling.

\section{CONCLUSION}

The presented AUV Abyss workflow and associated Jupyter Notebooks presented here summarize nearly a decade of deep sea high-resolution mapping experience and map production. Using the combination of careful mission planning and systematic post-processing make USBL navigation unnecessary and with this, increase the efficiency of ship time.
Jupyter Notebooks that provide a combination of live coding, scripting and narrative comments facilitate the use of MB-System for AUV MBES data processing. The automated documentation and guided archiving in the Jupyter Notebooks ensures an efficient map production and increased reproducibility. Combining MB-System with Jupyter Notebook enables adaptation of the workflow to other vehicles, or using multiple coordinated AUVs. The comprehensive documentation of the post-processing provides a first step towards automatization of AUV operations and MBES data processing.

\section{ACKNOWLEDGMENT}

Purchase of the AUV Abyss was funded by the German Research Foundation (DFG). It is currently operated by GEOMAR - Helmholtz Centre for Ocean Research Kiel, which also supported the development of the workflow and publishing this work. The EU-funded 'Blue Mining Project' and the BGR (Bundesanstalt für Geowissenschaften und Rohstoffe) INDEX project kindly provided the AUV data shown here, collected on board R/V Meteor (cruise M127; [5])and R/V Merian (INDEX2016-2) respectively.

\section{REFERENCES}

[1] GEOMAR Helmholtz-Zentrum für Ozeanforschung. (2016). Autonomous Underwater Vehicle „ABYSS“. Journal of large-scale research facilities, 2, A79. http://dx.doi.org/10.17815/jlsrf-2-149

[2] Haase, K., Koschinsky, A., Petersen, S., Devey, C., German, C., Lackschewitz, K., . . . Paulick, H. (2009). Diking, young volcanism and diffuse hydrothermal activity on the southern mid-atlantic ridge. $\begin{array}{llll}\text { Marine } \quad \text { Geology, } & \text { 266(1-4), } & 52 & -\end{array}$ http://dx.doi.org/10.1016/j.margeo.2009.07.012

[3] Yeo, I.A., Devey, C. W., LeBas, T. P., Augustin, N., \& Steinführer, A. (2016). Segment-scale volcanic episodicity: Evidence from the North Kolbeinsey Ridge, Atlantic. Earth and Planetary Science Letters, 439, 81 - 87. https://doi.org/10.1016/j.epsl.2016.01.029

[4] Escartín, J., et al. (2017). Tectonic structure, evolution, and the nature of oceanic core complexes and their detachment fault zones $\left(13^{\circ} 20^{\prime} \mathrm{N}\right.$ and $13^{\circ} 30^{\prime} \mathrm{N}$, Mid Atlantic Ridge). Geochemistry Geophysics Geosystems 18: 1451-1482. https://doi.org/10.1002/2016GC006775

[5] Petersen, S. (2017). Metal fluxes and resource potential at the slowspreading TAG mid-ocean ridge segment $\left(26^{\circ} \mathrm{N}, \mathrm{MAR}\right)-$ Blue Mining@ Sea - Cruise No. M127 - May 25 - June 28, 2016 Bridgetown (Barbados) - Ponta Delgada (Portugal). DFGSenatskommission für Ozeanographie, Bremen, 1-58. https://doi.org/10.2312/cr_m127

[6] Caress, D.W. (1999). MB-System - public domain software for processing swath mapping sonar data. Undersea Explorations.

[7] van Rossum, G. (1995). Python tutorial. Technical Report CS-R9526, Centrum voor Wiskunde en Informatica (CWI), Amsterdam.

[8] Kluyver, T., Ragan-Kelley, B., Pérez, F., Granger, B., Bussonnier, M., Frederic, J., Kelley, K., Hamrick, J., Grout, J., Corlay, S., Ivanov, P., Avila, D., Abdalla, S., Willing, C., Jupyter Development Team (2017). Jupyter Notebooks - a publishing format for reproducible computational workflows. Positioning and Power in Academic Publishing: Players, Agents and Agendas, $87-90$. http://dx.doi.org/10.3233/978-1-61499-649-1-87

[9] Wessel, P., Smith, W.H.F., Scharroo, R., Luis, J. F., and Wobbe, F. (2013). Generic Mapping Tools: Improved version released, EOS Trans. AGU, 94, 409-410. https://doi.org/10.1029/98EO00426

[10] https://git.geomar.de/auv-abyss/AUVAbyssWorkflow

[11] https://doi.org/10.3289/SW_1_2018

[12] Parker, R. L. (1994). Geophysical Inverse Theory. Princeton University Press, Princeton, NJ, 1994.

[13] Paull, L., Saeedi, S., Seto, M.L., \& Li, H. (2014). AUV Navigation and Localization: A Review. IEEE Journal of Oceanic Engineering, 39, 131-149. https://doi.org/10.1109/JOE.2013.2278891 